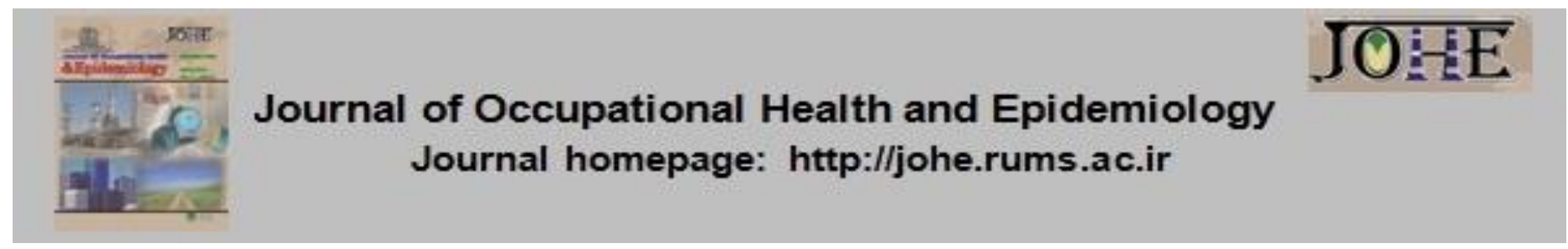

\title{
Needle stick and sharps injuries among healthcare workers in Fasa city, Southwestern Iran, 2017
}

\author{
Zhila Fereidouni ${ }^{1}$, Mehdi Amirkhani ${ }^{2}$, Javad Salami ${ }^{3}$, Majid Najafi Kalyani ${ }^{4^{*}}$ \\ 1- Assistant Prof., School of Nursing, Fasa University of Medical Sciences, Fasa, Iran. \\ 2- MSN, Instructor, School of Nursing, Fasa University of Medical Sciences, Fasa, Iran \\ 3- MSN, Lamerd Nursing School, Shiraz University of Medical Sciences, Shiraz, Iran \\ 4- Associate Prof., School of Nursing and Midwifery, Shiraz University of Medical Sciences, Shiraz, Iran
}

\section{Article Info}

* Corresponding author:

Majid Najafi Kalyani,

E-mail:

majidnajafi5@yahoo.com

\section{Article history}

Received: Jul, 2019

Accepted: Oct, 2019

10.29252/johe.8.3.156

Print ISSN: 2251-8096

Online ISSN: 2252-0902

Peer review under responsibility of Journal of Occupational Health and Epidemiology
Citation: Fereidouni Zh, Amirkhani M, Salami J, Najafi Kalyani M. Needle stick and sharps injuries among healthcare workers in Fasa city, Southwestern Iran, 2017. JOHE 2019; 8(3):156-62.

\section{Abstract}

Background:Among the most common occupational hazards threatening healthcare workers are needle stick injuries, injuries caused by sharp and cutting instruments, and contact with infectious pathogens transmitted by blood. This study aims to determine the prevalence and associated factors of needle stick injuries, injuries caused by sharp and cutting instruments, as well as blood and body fluids affecting healthcare workers.

Material and Methods: This research adopted a descriptive cross-sectional approach in which all healthcare workers at the two training hospitals affiliated with Fasa University of Medical Sciences were selected in 2017. After receiving ethical approval (IR.FUMS.REC.1396.243), the data collected by the researcher using a questionnaire and proportional sampling. The validity and reliability of the questionnaire were confirmed prior to use. Data analysis was performed by SPSS Statistics V22.0, using mean, frequency, and a Chi-Square test.

Results: According to the results of this study, $51.0 \%$ of the staff had experienced at least an injury caused by cutting tools, and $26.7 \%$ of them considered carelessness as the main cause of the injury. Nurses had the highest prevalence $(24.4 \%)$ of the experience among other staff.

Conclusions: The results imply that injuries caused by needles and sharp objects need more attention. In addition, improper reactions at the time of the injuries necessitate educating healthcare workers and increasing their knowledge about the dangers posed by these injuries

Keywords: Needle Stick Injuries, Healthcare Workers, Injuries, Hospital, Iran

\section{Introduction}

Medical staff working at hospitals are highly exposed to occupational hazards caused by daily exposure to body fluids and secretions $(1,2)$. Needle stick and sharp injuries are a major cause of infections among healthcare staff (3). Needle stick injuries significantly increase the transmission risk of the Human Immunodeficiency Virus (HIV) infection, hepatitis C and hepatitis B (3). Healthcare staff, such as physicians, nurses, health workers, and lab technicians are at risk more than others, and the prevalence of needle stick injuries is higher among them (4). Nurses are more than other healthcare workers at the risk of NSIs (5). It is estimated that about 800,000 needle stick injuries occur annually among healthcare workers in the United States (6). The annual prevalence of needle stick injuries in different states of the United States ranges from 14 to 839 cases per 1,000 persons working in healthcare environments (7). 
In a study conducted by Yung et al in Taiwan, the risk of sharp injuries was reported to be $50.1 \%$. However, the risk was lower in the staff with a shorter clinical history than those with a longer clinical history (8). The findings of a study in Turkey showed that $64 \%$ of the health center staff were at least once exposed to blood and body fluids during their professional occupation (9). Zeighami et al (2014) reported that the risk of needle stick and sharp injuries in the emergency room was three times more than the same risk in other sectors (10). In the study by Rezaei et al in Tehran, needle stick injuries and peripheral venous catheters were the most common types of occupational exposures. In addition, the highest prevalence of occupational exposure was observed at the time of venipuncture and syringe reinstalling (11).

Due to the economic, social, human, and health consequences of hospital incidents, they are of paramount importance, with the first step towards preventing and controlling them being to identify the prevalence rate and factors affecting their occurrence. Determining the frequencies and causes of such injuries as well as staff reactions to them would help adopt appropriate strategies to reduce their occurrence among healthcare workers. There has been no study conducted on the prevalence and causes of needle stick and sharp injuries in all classes of healthcare workers in Iran. Hence, given the importance of this issue and the necessity of safeguarding staff health, this study was conducted to determine the frequency and causes of needle stick and sharp injuries among healthcare workers at Fasa Hospital.

\section{Materials and Methods}

This study, with a descriptive cross-sectional approach, was conducted in 2017 at all training hospitals affiliated with Fasa University of Medical Sciences aimed at determining the frequencies and causes of needle stick and sharp injuries. The participants included all medical staff working at different sectors of the hospital, including emergency rooms, laboratories, operating rooms, as well as surgical, internal, and women wards, along with intensive care units. The ethics committee of Fasa University of Medical Sciences approved this study under code IR.FUMS.REC.1396.243. Written informed consent forms were obtained from all participants. The participants were selected based on proportional sampling from all wards of the hospital. The inclusion criteria consisted of having the working experience of at least one year at the hospital and being willing to participate in the research. Incomplete questionnaires were excluded from the analysis.

Data were collected using a researcher-made questionnaire containing two parts, with the first part being related to demographic information and the second part being connected with information about the location and place of the injury, the type of the harmful device, the activity causing the damage, and in general, measures taken after the injury. The content validity of the questionnaire was confirmed based on a review of the past research papers as well as comments provided by seven faculty members of Fasa University of Medical Sciences. The 'test retest' method was used to determine the reliability of the questionnaire. The correlation coefficient of the questionnaire was determined to be 0.9. The random proportional sampling method was employed, according to which the sample size was distributed among the two training centers of Shariati and Vali-e-Asr in Fasa city according to the number of employees. In addition, the sample size was allocated to each center in proportion to the number of employees. Samples were selected using staff lists and the simple random sampling method in each hospital ward. The questionnaires were completed by conducting face-to-face interviews with the selected individuals. Written informed consent forms were obtained from all participants. To observe ethical considerations and maintain confidentiality, the questionnaires were anonymous, and the information was collected confidentially in all stages of the study, having been kept by the researcher. Data were analyzed using SPSS Statistics V22.0 by descriptive statistics and a chi-square test.

\section{Results}

In this study, 176 participants were present, with 61 (34.7\%) and 115 (65.7\%) of whom having been male and female, respectively. The highest number of the participants in terms of the work experience was related to those with less than 5 years of experience (54.3\%), and the lowest number of the participants was related to those with the work experience of 21-25 years. Among the participants' occupations, the highest and lowest numbers belonged to the group of nurses and the medical emergency technicians with $24.4 \%$ and $1.7 \%$, respectively (Table 1 ). 
Table 1: Demographic features of the participants in this study

\begin{tabular}{llc}
\hline & Variable & \multicolumn{1}{c}{$\mathbf{N}(\%)$} \\
\hline \multirow{2}{*}{$\operatorname{sex}$} & Male & $61(34.7)$ \\
\hline & Female & $115(65.3)$ \\
\cline { 2 - 3 } & Physicians & $36(20.9)$ \\
\cline { 2 - 3 } Occupation & Nurses & $42(24.4)$ \\
& Operating room technicians & $25(14.5)$ \\
\cline { 2 - 3 } & Anesthesia technicians & $23(13.4)$ \\
\cline { 2 - 3 } & Paboratory technicians & $10(5.8)$ \\
\cline { 2 - 3 } & Emergency technicians & $4(2.3)$ \\
\cline { 2 - 3 }
\end{tabular}

In terms of the type of instruments causing injuries, surgical sutures were responsible for the highest amount of injuries (38.6\%), and syringes and laboratory tubes were responsible for the lowest amount of injuries (1.1\%). Among all the departments studied, the staff of the operating room department underwent the highest amount of injuries $(58.9 \%)$, and the staff of the departments of the intensive care unit (ICU), the cardiac care unit (CCU), the Post Coronary Care Unit (PCCU), and the maternity ward had the lowest amount of injuries (1.1\%) (Table 2).

Table 2: Factors causing needle stick and sharp injuries

\begin{tabular}{|c|c|c|}
\hline & Variable & $\mathbf{N}(\%)$ \\
\hline \multirow{7}{*}{ Setting } & Operation room & $63(70.0)$ \\
\hline & Emergency department & $13(14.4)$ \\
\hline & Injection unit & $1(1.1)$ \\
\hline & Laboratory & $2(2.2)$ \\
\hline & ICU & $1(1.1)$ \\
\hline & Maternity unit & $1(1.1)$ \\
\hline & CCU & $3(3.3)$ \\
\hline \multirow{7}{*}{ Instrument } & Syringe needle tips & $32(36.4)$ \\
\hline & Surgical sutures & $34(38.6)$ \\
\hline & PVC & $16(18.2)$ \\
\hline & Scalpels & $3(3.4)$ \\
\hline & Ampoules & $1(1.1)$ \\
\hline & Laboratory tubes & $1(1.1)$ \\
\hline & Surgery instruments & $1(1.1)$ \\
\hline \multirow{9}{*}{ Cause of injury } & Fatigue & $15(16.7)$ \\
\hline & Hurry & $21(23.3)$ \\
\hline & Stress & $2(2.2)$ \\
\hline & Carelessness & $24(26.7)$ \\
\hline & Inaccessibility of protective instruments & $4(4.4)$ \\
\hline & Colleague carelessness & $10(11.1)$ \\
\hline & Shortage of instruments & $1(1.1)$ \\
\hline & Wrong methods & $1(1.1)$ \\
\hline & Too much work & $12(13.3)$ \\
\hline \multirow{7}{*}{ Action taken } & Reporting to the supervisor & $6(6.7)$ \\
\hline & Washing with soap and water & $26(28.9)$ \\
\hline & Disinfection with disinfectants & $5(5.6)$ \\
\hline & Pressing injured sites & $11(12.2)$ \\
\hline & Sending patients' blood sample to the laboratory & $12(13.3)$ \\
\hline & Assessment of the HBS antibody & $2(2.2)$ \\
\hline & Nothing & $1(1.1)$ \\
\hline
\end{tabular}

In this study, $42 \%$ of injury cases occurred after patients had been treated, and the least injury cases were reported for the process of finding a vein $(3.4 \%)$. According to the results, carelessness, hurry, and fatigue were the most prevalent causes of injuries (66.7\%), and the adoption of wrong methods and the shortage of instruments were the least prevalent causes $(2.2 \%)$. Washing with soap and water and sending blood samples to the laboratory were performed in the majority of injury cases (58.9\%). In addition, in $2.2 \%$ of the cases no measures had been taken after the occurrence of injuries. Among the people who did not report an injury, the most common 
reason was the low probability of the infection $(37.5 \%)$, yet people were unacquainted with the reporting process in $2.1 \%$ of the cases. As to the site of the injuries, the highest and lowest amount of injuries occurred to hands (94.4\%) and eyes (5.6\%), respectively. Among all participants in the study, 155 participants were vaccinated, and 15 participants reported no vaccination experience.
According to Table 3, there has been a significant correlation between the type of occupations and the history of needle stick injuries. There was no significant correlation between the history of needle stick injuries and vaccination experience, gender, and information on the antibody titer (Table 3).

Table 3: The correlation between needle stick injuries and demographic variables

\begin{tabular}{ccccc}
\hline Variable & Sex & Occupation & History of vaccination & $\begin{array}{c}\text { Having information on the } \\
\text { antibody titer }\end{array}$ \\
\hline History of needle stick injuries & 0.598 & 0.001 & 0.064 & 0.546 \\
\hline
\end{tabular}

\section{Discussion}

Needle stick and sharp injuries as well as exposure to the fluid discharge of patients can lead to the transmission of highly dangerous diseases, which is considered as a risk factor by occupational therapists. According to the results of this study, among the total of 176 staff participating in the study, 90 individuals (51.12\%) experienced needle stick and sharp injuries, with this amount being less than that of the studies conducted by Azap in Turkey (9), Kermode in Australia (12), and Askarian in Shiraz (13). These differences could have been due to the type of healthcare workers, hospitals, and sample sizes. The aforementioned amount has been greater than that of the study conducted by Smith et al in Japan (14). A study conducted by Cho et al showed that $70 \%$ of nurses suffered needle stick and sharp injuries during the last year (15). Rais and Jamil reported in their study that $77 \%$ of the hospital staff experienced at least a needle stick injury (16). In the present study, all healthcare workers were evaluated in terms of NSIs, but in the aforementioned studies, only nurses were evaluated.

The total coverage rate of hepatitis $B$ vaccination (three times as standard protocol) was $90.9 \%$ in this study. According to a study carried out by Kathy Byrd et al, $69.5 \%$ of the hospital staff injected hepatitis $B$ vaccines in three phases, with this having been lower than the standard level (17). A study by Rosemary J. Burnett in South Africa reported that $67.9 \%$ of the staff received at least one dose of the hepatitis $B$ vaccine. In the aforementioned study, only $19.9 \%$ of the staff were fully vaccinated (18). These differences could be attributed to different protocols used for the vaccination of healthcare staff at hospitals.

In this study, the highest degree of damage was reported to have been occurred to the staffin the operating room, which is consistent with the results of the study conducted by Serfafiska in Poland
(19). This could have been due to the nature of the procedure and the risks present in the operation room. The staff of the operating room have to press the needle into the patient's body and remove it several times, with the duration of this procedure being longer than other injections; thus, the chance of injuries is higher. The results of the study by Cho et al showed that high emotional exhaustion as well as working at surgical and operating rooms were associated with an increase in the chance of needle stick injuries (15). The results of the study by Adib Haj Bagheri showed that the majority of needle stick and sharp injuries occurred to the operating room staff (20). According to the results of the study by Martin et al, $64.5 \%$ of the staff had the history of at least one injury during the past five years. The most affected people were reported to be nurses and women (21). A study by Nagao et al showed that $72.2 \%$ of the injured staff were nurses (22). In the same vein, the results of the study by Yushikawa showed that the incidence of injuries was 3.2 times higher in nurses than in other staff. Most injuries occurred in the operating room and patient rooms (23). The results of the present study are consistent with those of the above-mentioned studies, thereby implying that nurses are more at the risk of NSIs due to the nature of their works.

In the present study, most of the damage occurred after injections and before disposing of the contaminated equipment; likewise, the results of a study in Shiraz attributed the greater amount of damage to intravenous injections (24). In the study by Damet et al, the highest extent of damage was reported to have happened when removing the needle cover. In most studies, insufficient experience, rush, and fatigue have been mentioned as the causes of injuries (25). In Nsubuga's study, the most prevalent risk factors for needle stick injuries included not attending training sessions, being a nurse, working at the 
surgery department, having less than 10 years of work experience, working for long hours, needle re-closure, and not using gloves (26). In the study of Rais, $40.3 \%$ of the injuries occurred while using needles, and $58.4 \%$ of the injuries occurred when removing syringes. In $72.7 \%$ of the cases, fingers were reported to have been damaged (16). This finding has been consistent with a meta-analysis conducted by Fereidouni et al (3), in which they confirmed that injections were the most common causes of occupational injuries in healthcare workers.

The most common cause of injuries in nurses was the re-closure of syringes (39.1\%). In the study by KuruZuum et al (27) and Yushikawa et al, (23) disposable syringes and suture needles were the most common causes of injuries. The results of the study by Vos et al showed that needle tips were the damaging items in $53 \%$ of the cases, with the wrong disposal and re-closuring of the needles reported as the two common causes of injuries (28). The results of the study by $\mathrm{Bi}$ et al showed that hollow needles were responsible for $51.7 \%$ of injuries. In another study, $40.4 \%$ of the injuries were reported to happen when using tools, $27.1 \%$ of the injuries after use and before disposal, $11.3 \%$ of the injuries during the disposal process, and $8.9 \%$ of the injuries at the time of cleaning and collecting the tools (29). In the study by Nagao et al, $55.9 \%$ of the injuries occurred after the use of needles and before their disposal (22). The results of the present study are consistent with those of the above-mentioned studies, implying that recapping is the most common activity that causes NSIs.

In the present study, medical staff were reported to have been responsible for $6.7 \%$ of injury cases. The most likely reason for this could be the low risk of infections and dissatisfaction with the lack of follow-ups, having been consistent with the results of the study by Askarian (13). A study by Laishram et al reported that $43 \%$ of the staff did not report their injuries (30). In the study by Castigliola on nurses, it was reported that imagine the source of the injury being safe was the major reason for the failure to report needle stick injuries (31). In the study by Gershon et al, $45.8 \%$ of the injuries were not reported (32). In the study by Elmiyeh et al, not understanding the transmission risk of infectious diseases was reported as the major reason for not reporting injuries (33). The reason for the low rate of reporting injuries by the healthcare staff in the present study is different with that of other studies, which could be attributed to the possible lack of a clear process for reporting NSIs by healthcare staff.
The results of this study showed that carelessness, rush, fatigue, and high workloads were the most common causes of injuries. The results of the study by Cho et al showed that fatigue and workloads were associated with an increase in the chance of needle stick injuries (15). According to the study by Elmiyeh et al, heavy workloads and being too busy were the major causes of needle stick injuries (33).

According to the present study, there was a significant correlation between the type of occupation and the history of needle stick injuries. In the present study, the most common individuals associated with needle stick injuries were nurses and operating room technicians. The results of a systematic review study by Fereidouni et al showed that nurses were the most common healthcare staff associated with needle stick injuries (2). This could be due to the nature of nursing and the direct care of patients.

\section{Conclusion}

To sum it up, the results of this study showed that more than half of the participants (51.12\%) suffered from needle stick and sharp injuries. Furthermore, carelessness, hurry, and fatigue were the most common causes of injuries (66.7\%). The risk of getting injured by sharp devices seems to be serious at hospitals. Therefore, it is recommended to use skin and mucous protectors as well as gloves when working with sharp tools to reduce the transmission risk of pathogens from patients to medical staff. Defining and explaining instructions for following relevant stages after injuries and erecting them on the walls of different departments, holding different training classes, increasing the number of employees in different departments based on global standards to avoid an increase in workloads and fatigue, and avoiding the reduction of one's focus when working with sharp devices and needles could be very helpful in reducing damage to different parts of the body. Health policymakers and planners could use the results of this study for the prevention of NSIs. This study had two potential limitations; firstly, it was conducted at two training hospitals; secondly, the self-made questionnaire was distributed among participants.

\section{Acknowledgement}

This article was extracted from an approved research project, with the Grant Number of 95204, by Fasa University of Medical Sciences, Fasa City, Iran. We would like to thank the research deputy of Fasa University of Medical Sciences for offering support to this project. The authors would also like 
to thank Shiraz University of Medical Sciences (Shiraz, Iran), the Center for Clinical Research Development at Nemazee Hospital, and Dr. NasrinShokrpour for offering valuable editorial assistance.

\section{Conflict of interest: None declared.}

\section{References}

1. Azmoon $\mathrm{H}$, Dehghan $\mathrm{H}$, Pourabdian $\mathrm{S}$. Evaluation of trait anxiety levels between exposed and non-exposed health care workers to needle stick in Isfahan Al-Zahra hospital. Pak J Med Sci 2013; 29((1) Suppl):351-3.

2. Fereidouni Z, Kameli Morandini M, Dehghan A, Jamshidi N, Najafi Kalyani M. The Prevalence of Needlestick Injuries and Exposure to Blood and Body Fluids among Iranian Healthcare Workers: A Systematic Review. International Journal of Medical Reviews 2018; 5(1):35-40.

3. Fereidouni Z, Dehghan A, Najafi Kalyani M. Common Causes Associated with Occupational Injuries in Iranian Health Care Workers: A Systematic Review and Meta-Analysis Study. International Journal of Medical Reviews 2018; 5(2):77-81.

4. Huang SL, Lu Q, Fan SH, Zong ZY, Hou TY, Chen BY, et al. Sharp instrument injuries among hospital healthcare workers in mainland China: a cross-sectional study. BMJ Open 2017; 7(9):e017761.

5. Lori JR, McCullagh MC, Krueger A, Oteng R. Sharps injuries among emergency department nurses in one tertiary care hospital in Ghana. Int Emerg Nurs 2016; 28:14-9.

6. Pruss-Ustun A, Rapiti E, Hutin Y. Estimation of the global burden of disease attributable to contaminated sharps injuries among health-care workers. Am J Ind Med 2005; 48(6):482-90.

7. Lee JM, Botteman MF, Xanthakos N, Nicklasson L. Needlestick injuries in the United States. Epidemiologic, economic, and quality of life issues. AAOHN J 2005; 53(3):117-33.

8. Yang Yh, Wu MT, Ho CK, Chuang HY, Chen L, Yang CY, et al. Needlestick/sharps injuries among vocational school nursing students in southern Taiwan. Am J Infect Control 2004; 32(8):431-5.

9. Azap A, Ergonul O, Memikoglu KO, Yesilkaya A, Altunsoy A, Bozkurt GY, et al. Occupational exposure to blood and body fluids among health care workers in Ankara, Turkey. Am J Infect Control 2005; 33(1):48-52.

10. Zeighami R, Azimian J, Haghi M, Kaboodi B, Bijani B, Haghi M. A comparison between the risk of needle stick injuries among nurses in emergency wards and nurses in other wards of hospitals. Modern Care Journal 2013; 10(4):272-8.

11. Rezaei Sh , Rabirad N, Tamizi Z , Fallahi Khoshknab M , Mohammad Nejad E , Mahmoodi M. Needle sticks injuries among heath care workers in emergency medical centers in Tehran University of Medical Sciences Hospitals (2007-2010). Journal of Health Promotion Management 2012; 1(3):4654.

12. Kermode $M$, Jolley $D$, Langkham $B$, Thomas MS, Crofts N. Occupational exposure to blood and risk of bloodborne virus infection among health care workers in rural north Indian health care settings. Am J Infect Control 2005; 33(1):34-41.

13. Askarian M, Malekmakan L. The Frequency of Needlestick and Sharps Injuries during Clinical Training Setting in Dental Students of Shiraz University of Medical Sciences in 2005. Journal Kerman University of Medical Sciences 2005; 12(4): 265-70

14. Smith DR, Mihashi M, Adachi $Y$, Nakashima $Y$, Ishitake T. Epidemiology of needlestick and sharps injuries among nurses in a Japanese teaching hospital. J Hosp Infect 2006; 64(1):449.

15. Cho E, Lee H, Choi M, Park SH, Yoo IY, Aiken LH. Factors associated with needle stick and sharp injuries among hospital nurses: A crosssectional questionnaire survey. Int J Nurs Stud 2013; 50(8):1025-32.

16. Rais N, Jamil HM. Prevalence of needle stick injuries among health care providers. International Journal of Endorsing Health Science Research 2013; 1(2):73-9.

17. Byrd KK, Lu PJ, Murphy TV. Hepatitis B vaccination coverage among health-care personnel in the United States. Public Health Rep 2013; 128(6):498-509.

18. Burnett RJ, François G, Mphahlele MJ, Mureithi JG, Africa PN, Satekge MM, et al. Hepatitis $B$ vaccination coverage in healthcare workers in Gauteng Province, South Africa. Vaccine 2011; 29(25):4293-7.

19. Serafinska S, Smolinski P, Gladysz A. Critical evaluation of reporting on postexposure skin damage incidents and its consequences for Polish health workers. Med Pr 2006; 57(5):43950.

20. Adib-Hajbegheri M, Lotfi MS. Behavior of healthcare workers after injuries from sharp instruments. Trauma Mon 2013; 18(2):75-80.

21. Martins A, Coelho AC, Vieira M, Matos M, Pinto $M L$. Age and years in practice as factors associated with needlestick and sharps injuries among health care workers in Portuguese hospitals. Accid Anal Prev 2012; 47:11-5.

22. Nagao $Y$, Baba $H$, Torii $K$, Nagao $M$, Hatakeyama K, Linuma $Y$, et al. A long-term study of sharp injuries among health care workers in Japan. Am J Infec Control 2007; 35(6):407-11.

23. Yushikawa T, Wada K, Lee JJ, Mitsuda T, Kidouchi $\mathrm{K}$, Kurosu $\mathrm{H}$, et al. Incidence rate of needlestick and sharps injuries in 67 Japanese hospitals: a national surveillance study. PLoS One 2013; 8(10):e77524. 
24. Norsayani MY, Noor Hassim I. Study of incidence of needle stick injury and factors associated with this problem among medical students. J Occup Health 2003; 45(3):172-8.

25. Dement JM, Epling C, Ostbye T, Pompeil LA, Hunt DL. Blood and body fluid exposure risk among health care workers: results from the Duke Health and Safety Surveillance System. Am J Ind Med 2004; 46(6):637-48.

26. Nsubuga FM, Jaakkola MS. Needle sticks injuries among nurses in sub-Saharan Africa. Trop Med Int Health 2005; 10(8):773-81.

27. Kuruuzum Z, Yapar N, Avkan-Oguz V, Aslan H, Ozbek OA, Cakir N, et al. Risk of infection in health care workers following occupational exposure to a noninfectious or unknown source. Am J Infect Control 2008; 36(10):e27-31.

28. Vos D, Gotz HM, Richardus JH. Needlestick injury and accidental exposure to blood: the need for improving the hepatitis $B$ vaccination grade among health care workers outside the hospital. Am J Infect Control 2006; 34(9):610-2.

29. Bi P, Tully P, Pearce S, Hiller JE. Occupational blood and body fluid exposure in an Australian teaching hospital. Epidemiol Infect 2006; 134(3):465-71

30. Laishram J, Keisam A, Phesao E, Tarao MSh, Laloo VJ, Devi HS. Prevalence of needle sticks injuries among nurses in a tertiary care hospital and their immediate response. Int $\mathrm{J}$ Med Public Health 2013; 3(4):257-60.

31. Castigliola V, Frid A, Letondeur C, Strauss K. Needlestick injuries in European nurses in diabetes. Diabetes Metab 2012; 38(1):S9-14.

32. Gershon RR, Pearson JM, Sherman MF, Samar SM, Canton AN, Stone PW. The prevalence and risk factors for percutaneous injuries in registered nurses in the home health care sector. Am J Infect Control 2009; 37(7):525-33.

33. Elmiyeh B, Whitaker S, James MJ, Chahal CA, Galea A, Alshafi K. Needle-stick injuries in the national health service: a culture of silence. J R Soc Med 2004; 97(7):326-7. 